\title{
Upper Respiratory Tract Microbiome of Australian Aboriginal and Torres Strait Islander Children in Ear and Nose Health and Disease
}

\author{
Andrea Coleman, ${ }^{\text {a,b }}$ Julian Zaugg, ${ }^{c}$ Amanda Wood, ${ }^{d}$ Kyra Cottrell, ${ }^{a}$ Eva Grahn Håkansson, ${ }^{e}$ Jasmyn Adams, ${ }^{d}$ Matthew Brown, ${ }^{d}$ \\ Anders Cervin, ${ }^{\text {a,f }}$ (D) Seweryn Bialasiewicz ${ }^{c, g}$
}

\author{
aThe University of Queensland Centre for Clinical Research, Herston, Australia \\ bTownsville University Hospital, Townsville, Australia \\ cAustralian Centre for Ecogenomics, The University of Queensland, St. Lucia, Australia \\ ¿Queensland Health Deadly Ears Program, Brisbane, Australia \\ eClinical Microbiology, Umeå University, Umeå, Sweden \\ fRoyal Brisbane and Women's Hospital, Brisbane, Australia \\ gQueensland Paediatric Infectious Diseases Laboratory, Queensland Children's Hospital, South Brisbane, Australia
}

ABSTRACT The objective of this study was to examine the nasal microbiota in relation to otitis media (OM) status and nose health in Indigenous Australian children. Children 2 to 7 years of age were recruited from two northern Australian (Queensland) communities. Clinical histories were obtained through parent interviews and reviews of the medical records. Nasal cavity swab samples were obtained, and the children's ears, nose, and throat were examined. DNA was extracted and analyzed by $16 \mathrm{~S}$ rRNA amplicon next-generation sequencing of the V3/N4 region, in combination with previously generated culture data. A total of 103 children were recruited (mean age, 4.7 years); 17 (16.8\%) were healthy, i.e., normal examination results and no history of OM. The nasal microbiota differed significantly in relation to OM status and nose health. Children with historical OM had greater relative abundance of Moraxella, compared to healthy children, despite both having healthy ears at the time of swabbing. Children with healthy noses had greater relative abundance of Staphylococcus aureus, compared to those with rhinorrhea. Dolosigranulum was correlated with Corynebacterium in healthy children. Haemophilus and Streptococcus were correlated across phenotypes. Ornithobacterium was absent or was present with low relative abundance in healthy children and clustered around otopathogens. It correlated with Helcococcus and Dichelobacter. Dolosigranulum and Corynebacterium form a synergism that promotes upper respiratory tract (URT)/ear health in Indigenous Australian children. Ornithobacterium likely represents "Candidatus Ornithobacterium hominis" and in this population is correlated with a novel bacterium that appears to be related to poor URT/ear health.

IMPORTANCE Recurring and chronic infections of the ear (OM) are disproportionately prevalent in disadvantaged communities across the globe and, in particular, within Indigenous communities. Despite numerous intervention strategies, OM persists as a major health issue and is the leading cause of preventable hearing loss. In disadvantaged communities, this hearing loss is associated with negative educational and social development outcomes, and consequently, poorer employment prospects and increased contact with the justice system in adulthood. Thus, a better understanding of the microbial ecology is needed in order to identify new targets to treat, as well as to prevent the infections. This study used a powerful combination of 16S rRNA gene sequencing and extended culturomics to show that Dolosigranulum pigrum, a bacterium previously identified as a candidate protective species, may require cocolonization with
Citation Coleman A, Zaugg J, Wood A, Cottrell K, Håkansson EG, Adams J, Brown M, Cervin A, Bialasiewicz S. 2021. Upper respiratory tract microbiome of Australian Aboriginal and Torres Strait Islander children in ear and nose health and disease. Microbiol Spectr 9:e00367-21. https://doi.org/10.1128/Spectrum.00367-21. Editor Aude A. Ferran, INTHERES

Copyright $\odot 2021$ Coleman et al. This is an open-access article distributed under the terms of the Creative Commons Attribution 4.0 International license.

Address correspondence to Andrea Coleman, a.coleman2@uq.edu.au, or Seweryn Bialasiewicz, seweryn@uq.edu.au.

Received 23 May 2021

Accepted 9 September 2021

Published 20 October 2021 
Corynebacterium pseudodiphtheriticum in order to prevent OM. Additionally, emerging and potentially novel pathogens and bacteria were identified.

KEYWORDS $16 \mathrm{~S}$ rRNA, Aboriginal and Torres Strait Islander, Corynebacterium, Dolosigranulum, Indigenous, Nose, Ornithobacterium, otitis media, ecology, microbiome

titis media (OM), an inflammation/infection of the middle ear, is a common pediatric condition (1). In many Indigenous populations globally, however, there is a disproportionately large OM-associated burden, negatively affecting schooling and employment outcomes $(1,2)$. Previous microbiological studies related to $\mathrm{OM}$ in Indigenous populations were largely limited to the main otopathogens (Streptococcus pneumoniae, Haemophilus influenzae, and Moraxella catarrhalis), using culture-dependent methods, and seldom included healthy Indigenous control children (3). One study used 16S rRNA next-generation sequencing (NGS) to explore the middle ear effusion and nasopharyngeal/adenoid microbiota in relation to OM with effusion (OME) in 11 Aboriginal and/or Torres Strait Islander (referred to as Indigenous Australian) children (4), which confirmed the association of otopathogen-containing genera and OME.

We previously used culturomics and species-specific quantitative PCR (qPCR) to explore the nasal microbiota in relation to ear health and $\mathrm{OM}$ in 103 Indigenous Australian children (5). We found that children with historical or current OM/upper respiratory tract (URT) infection had large otopathogen loads and higher rates of detection of rhinovirus (5). In contrast, Corynebacterium pseudodiphtheriticum and Dolosigranulum pigrum were associated with URT/ear health (5). However, culturebased analyses can be insensitive to microbial population structure and fastidious or unculturable organisms, such as the recently described "Candidatus Ornithobacterium hominis" $(6,7)$. To address this limitation, 165 rRNA NGS, supplemented with the existing culturomics data, was used to investigate the broader bacterial microbiome and how it relates to ear and nose health and disease in Indigenous Australian children.

\section{RESULTS}

Patients and samples. In total, 103 children were recruited; 2 children refused swabbing, resulting in 101 swabs for analysis. All swabs met quality assurance criteria for endogenous retrovirus 3 (ERV3) testing (8). Raw sample 16S rRNA read depth ranged from 149× to $262,880 \times$ (median, $119,693 \times$ ), with quality, contamination, and nonspecific filtering resulting in the remaining read depth ranging from $0 \times$ to $163,794 \times$ (median, $66,929 \times$ ). Fourteen samples were subsequently excluded because they did not meet quality control criteria. The agreement between culturomics and $16 \mathrm{~S}$ rRNA gene sequencing was $59.2 \%$, with a Cohen's kappa value of 0.08 . The low level of agreement was predominately due to the high sensitivity of detection by $16 \mathrm{~S}$ rRNA gene sequencing, which detected an average of 14.3 more genera per sample (range, 1 to 73 genera per sample), compared to culture (means of 18.0 and 3.7 genera per sample, respectively).

Nasal microbiota in relation to ear health. Only 17 children (16.8\%) had no history of OM and normal ear, nose, and throat (ENT) examination results at the time of swabbing (never OM), 7 (6.9\%) had a perforated tympanic membrane (TM), 18 (17.8\%) had middle ear effusion, 4 (4.0\%) had acute OM (AOM), and 55 had a history of OM (HxOM) but a normal TM at the time of swabbing (54.5\%) (Table 1). Due to small numbers, AOM samples were excluded from further analyses. There was a significant difference in the nasal microbiota in relation to OM status (permutational multivariate analysis of variance [PERMANOVA], $F=2.101, P=0.0027)$, although with dispersion differences (analysis of multivariate homogeneity [PERMDISP], $F=3.341, P=0.0244$ ). Among children who had healthy TMs at the time of sampling, the HxOM group had greater mean abundance of Moraxella, compared to the never-OM group (31.22\% versus $20.22 \%$; $P<0.05$ ) (Fig. 1; also see Table S1 in the supplemental material). The relative abundance of nine Dolosigranulum amplicon sequence variants (ASVs) differed significantly in relation to OM status; ASVs 588 and 2067 were more abundant in children with normal TMs, while ASVs 1030, 1069, and 1528 were more abundant in children with OM 
TABLE 1 Demographic and clinical details of participants

\begin{tabular}{|c|c|c|c|}
\hline \multirow[b]{2}{*}{ Characteristic } & \multicolumn{2}{|l|}{ Data for: } & \multirow{2}{*}{$\begin{array}{l}P \text { for difference between } \\
\text { remote and rural communitie }\end{array}$} \\
\hline & Remote community ( $n=59$ ) & Rural community ( $n=44)$ & \\
\hline$\overline{\text { Female gender (no. [\%]) }}$ & $33(47.7)$ & $21(47.7)$ & 0.41 \\
\hline Age (mean $\pm \mathrm{SD})(\mathrm{mo})$ & $57.0 \pm 13.4$ & $55.4 \pm 18.6$ & 0.61 \\
\hline Educational attendance (no. [\%]) & & & $<0.001$ \\
\hline School & $5(8.5)$ & $14(31.8)$ & \\
\hline Preschool & $43(72.9)$ & $11(25.0)$ & \\
\hline Daycare & $5(8.5)$ & $18(40.9)$ & \\
\hline Home & $6(10.2)$ & $1(2.3)$ & \\
\hline No. of people in the home (mean $\pm S D$ ) & $5.8 \pm 2.2$ & $4.9 \pm 1.6$ & 0.04 \\
\hline Pneumococcal vaccination $(\text { no. [\%] })^{a}$ & $56(94.9)$ & $37(84.1)$ & 0.04 \\
\hline HxOM group (no. [\%]) & $51(86.4)$ & $25(56.8)$ & $<0.001$ \\
\hline Never-OM group (no. [\%]) & $5(8.5)$ & $12(27.3)$ & 0.01 \\
\hline Historical type of OM (no. [\%]) & & & 0.07 \\
\hline AOM & $25(42.4)$ & $18(40.9)$ & \\
\hline AOM with perforation & $6(10.2)$ & $2(8.0)$ & \\
\hline OME & $3(5.1)$ & $1(4.0)$ & \\
\hline Chronic suppurative OM & $14(23.7)$ & $1(4.0)$ & \\
\hline Unknown & $3(5.1)$ & $3(12.0)$ & \\
\hline Otoscopic finding at sampling (no. [\%]) & & & 0.24 \\
\hline Bilateral normal TM & $26(44.1)$ & $29(65.9)$ & \\
\hline Effusion & $13(22.3)$ & $5(11.4)$ & \\
\hline AOM & $2(3.4)$ & $2(4.5)$ & \\
\hline Perforation & $5(8.5)$ & $2(4.5)$ & \\
\hline Unable to visualize TM & $13(22.0)$ & $6(13.6)$ & \\
\hline Nasal discharge at sampling (no. [\%]) & & & 0.01 \\
\hline None & $30(50.8)$ & $35(79.5)$ & \\
\hline Serous & $10(16.9)$ & $3(6.8)$ & \\
\hline Purulent & $19(32.2)$ & $6(13.6)$ & \\
\hline Oropharynx at sampling (no. [\%]) & & & 0.73 \\
\hline Tonsillitis & 0 & 0 & \\
\hline Pharyngitis & $2(3.4)$ & $1(2.3)$ & \\
\hline Season of collection (no. [\%]) & & & 0.01 \\
\hline Winter & $7(11.9)$ & 0 & \\
\hline Spring & $29(49.2)$ & $16(36.4)$ & \\
\hline Summer & 0 & 0 & \\
\hline Autumn & $23(38.9)$ & $28(63.6)$ & \\
\hline
\end{tabular}

${ }^{a}$ According to the Australian Vaccination Schedule (22).

(see Table S2) The relative abundance of Dolosigranulum was positively correlated with that of Corynebacterium in the never-OM group and those of both Corynebacterium and Moraxella in the HxOM group; there was no significant correlation between Dolosigranulum and the other main otopathogen-containing genera (see Fig. S1). Children with effusion had greater mean relative abundance of Ornithobacterium (34.1\%), compared to the never-OM group (28.4\%); although this was nonsignificant according to DESeq2, it was significant according to Dunn's test (adjusted $P=0.018$; Kruskal-Wallis test, $P=0.021$ ) (see Fig. S2).

Network analyses showed that taxon correlations largely differed according to OM status, with the notable exception of Streptococcus and Haemophilus, which were correlated across all groups. Never-OM children had a more complex network of correlated genera, compared to the HxOM group, despite both groups having normal TMs at the time of swabbing (Fig. 2). Dolosigranulum was positively correlated with different genera across all OM phenotypes, with exception of the effusion group, with Corynebacterium in the never-OM group, and with Moraxella and Neisseriaceae in the $\mathrm{HxOM}$ and TM perforation groups, respectively (Fig. 2). Our culturomic data suggested that the species representing the associated genera were Dolosigranulum pigrum and 


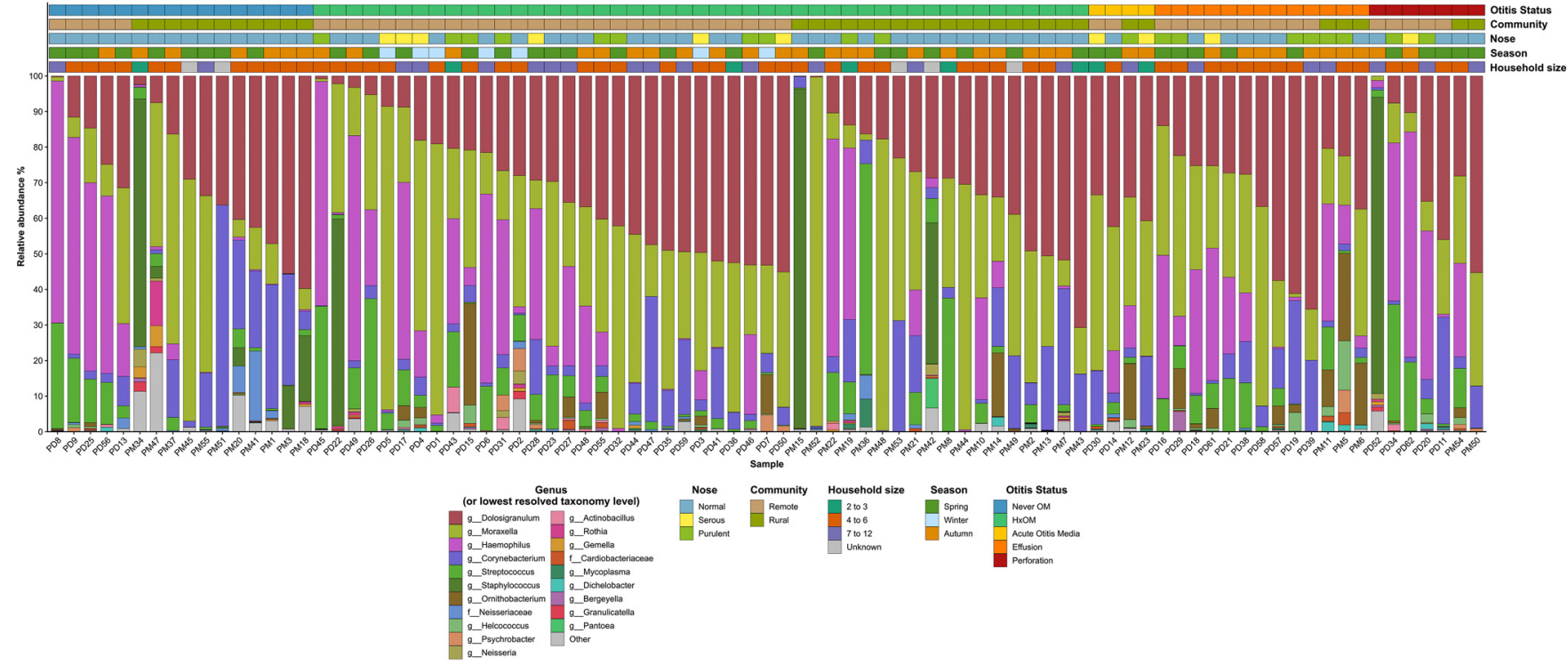

FIG 1 Mean relative microbial abundances of the 20 most abundant genera (or lowest resolved taxonomic level) across all samples, illustrating differences in OM status, community of residence, and other key variables. Microbes with lower abundances have been combined as "other" (gray). To improve interpretability, samples have been ordered by OM status, community, and Dolosigranulum abundance.

Corynebacterium pseudodiphtheriticum (5). Ornithobacterium was correlated with Helcococcus and Dichelobacter (Fig. 2). There were no significant differences in alpha diversity in relation to OM status (see Table S3).

Nasal microbiota in relation to nose health. The nasal microbiota was significantly related to nose health (PERMANOVA, $P<0.001, F=2.98$; PERMDISP, $F=2.753$, $P=0.068$ ). Compared to purulent rhinorrhea, children with healthy noses had significantly greater mean relative abundance of Staphylococcus (6.68\% versus $0.004 \%$ ) and Neisseriaceae $(0.868 \%$ versus $0.096 \%$ ) (all $P<0.001$ ) (Fig. 1 ; also see Table S2). ASV analysis showed that Staphylococcus aureus likely accounted for the Staphylococcus detections. Similar to ear health, multiple Dolosigranulum ASVs were detected across nose phenotypes (see Table S2). Network complexity and correlation patterns between Dolosigranulum and other bacteria in relation to nose status were similar to those seen within OM status (Fig. 3). Staphylococcus was correlated negatively with Moraxella in healthy noses; however, Ornithobacterium was present in all phenotypes and was correlated with Helcococcus, Dichelobacter, and Cardiobacteriaceae (Fig. 3). There were no significant differences in alpha diversity in relation to nose health (see Table S3).

Nasal microbiota in relation to season, household occupancy, and community. No relationship was found between nasal microbiota and season (PERMANOVA, $P=0.456$, $F=1.00$; PERMDISP, $P=0.192, F=1.619$ ) or household occupancy (PERMANOVA, $P=0.748$, $F=0.791$; PERMDISP, $P=0.844, F=0.181$ ). There were no significant differences in relative abundance or alpha diversity for these variables (Fig. 1; also see Table S3). The nasal microbiota differed significantly in relation to community of residence (PERMANOVA, $P<0.001$, $F=3.71$ ), although with dispersion differences (PERMDISP, $F=7.87, P=0.005$ ). No separation was observed between the two communities in principal-component analysis (PCA) (Fig. 4).

\section{DISCUSSION}

We demonstrated that the nasal microbiota of Indigenous Australian children was related to ear and nose health. Healthy children with no history of OM showed a relationship between Dolosigranulum and Corynebacterium. We detected Ornithobacterium in children with $\mathrm{OM}$, suggesting a potential role as a novel otopathogen in this population.

In relation to OM status, Moraxella had a greater relative abundance in $\mathrm{HxOM}$ children, compared to children with no history of OM, despite both groups having healthy ears at the time of swabbing. In children with healthy noses, there was a negative correlation 


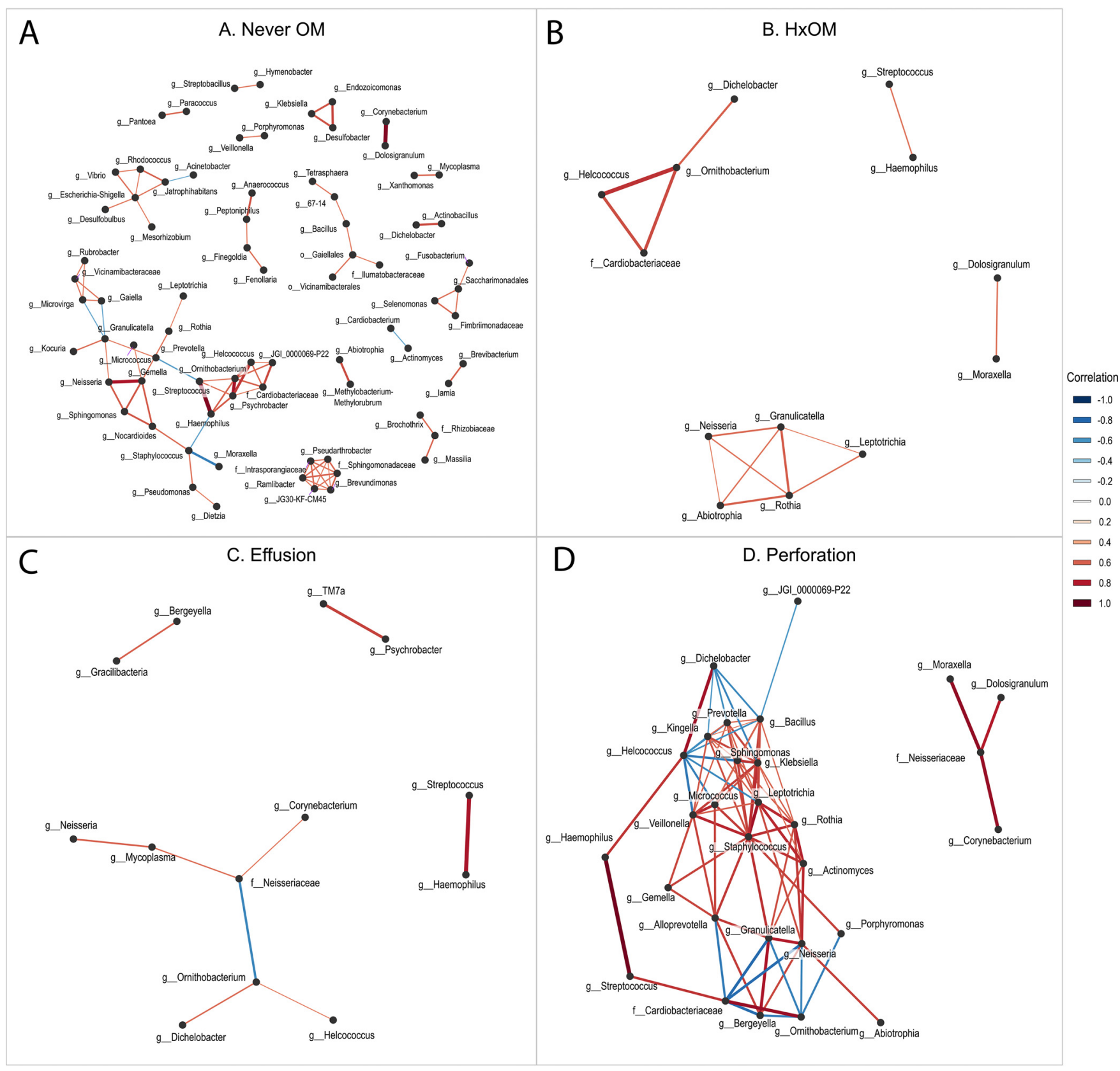

FIG 2 Network correlation analysis, showing differences in relationships of genera in the context of OM status. (A) Never-OM group. (B) HxOM group. (C) Middle ear effusion group. (D) TM perforation group. Connections between genera indicate significant correlation $(P$ value of $\leq 0.05$ and absolute correlation of $\geq 0.5$ ) between their respective abundances. The strength and direction of correlations are indicated by line thickness and color, respectively (e.g., Dolosigranulum and Corynebacterium show very strong positive correlation in the never-OM group). Genera are labeled by their lowest resolved taxonomic level.

between Moraxella and Staphylococcus. Moraxella strains are common nasal colonizers whose abundance can increase during acute respiratory infections, leading to prolonged periods of enrichment within the nasal microbiota $(9,10)$. Thus, the observed increase in Moraxella in $\mathrm{HxOM}$ children may be a downstream persistent effect of past respiratory infections (e.g., OM), leading to a remodeled microbiome distinct from that of children who did not contract OM. In vitro studies demonstrated that some Staphylococcus species can inhibit the growth of $M$. catarrhalis (11), which may account for their negative correlation within healthy noses in our cohort.

A combination of 16 S rRNA NGS and culturomic data strongly suggested that correlations exist between $C$. pseudodiphtheriticum and D. pigrum in healthy children with 


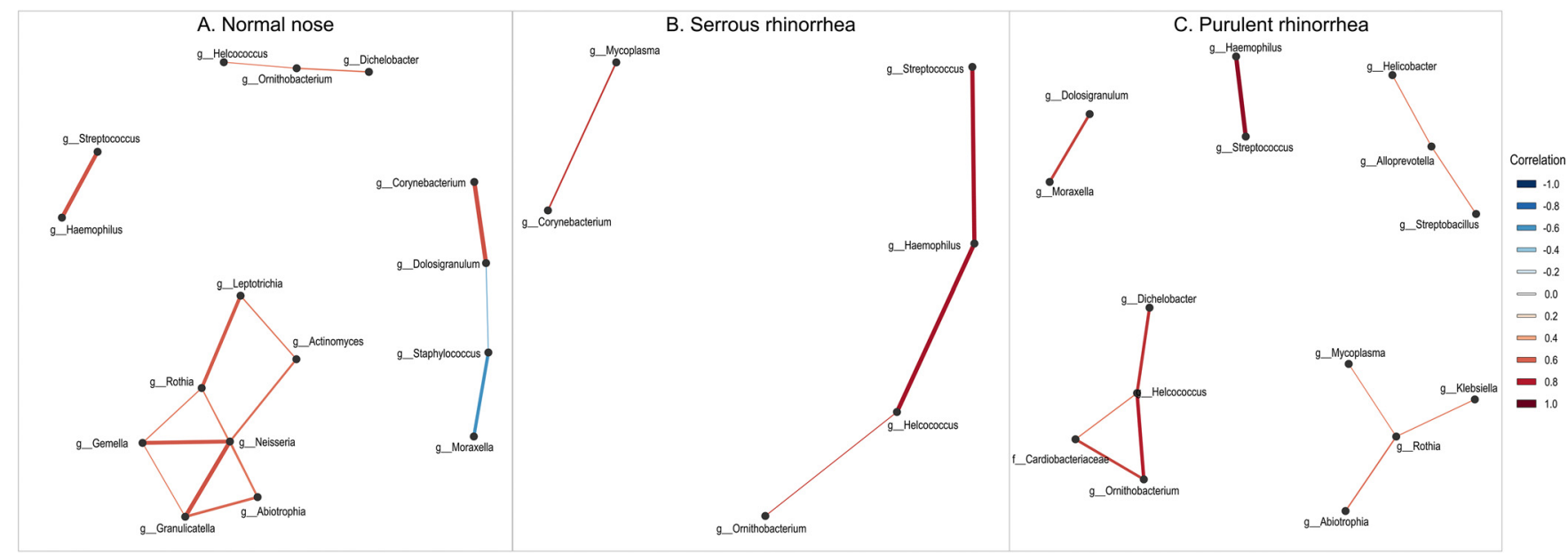

FIG 3 Correlation network analysis of genera in relation to nose health, showing differential Dolosigranulum relationships. Connections between genera indicate significant correlation ( $P$ value of $\leq 0.05$ and absolute correlation of $\geq 0.5$ ) between their respective abundances. The strength and direction of correlations are indicated by line thickness and color, respectively (e.g., Streptococcus and Haemophilus show very strong positive correlation in all nose status groups but Haemophilus has an additional strong correlation with Helcococcus only in the group of children with serous rhinorrhea). Genera are labeled by their lowest resolved taxonomic level.

no rhinorrhea and no historical OM. In non-Indigenous infants, Corynebacterium and Dolosigranulum are well recognized as being associated with a stable nasopharyngeal microbiota, conferring URT and ear health (10, 12-15). In vitro studies of CorynebacteriumDolosigranulum relationships demonstrated complex interactions that were species specific, which may be dependent on the use of host resources (16). However, both C. pseudodiphtheriticum and D. pigrum were required for the inhibition of S. pneumoniae; neither species could inhibit the growth of S. pneumoniae alone (16). These in vitro findings corroborate our in vivo data and warrant further investigation, particularly with the view toward prevention/control of otopathogen colonization in the nose and consequent ear health benefits.

Dolosigranulum was ubiquitous in the nasal microbiota of our population; however, examination at the level of ASVs suggests that this may be a heterogeneous group. The method of ASV analysis has greater precision than analysis of operational taxonomic units (OTUs), which was used in prior URT microbiota research, and thus may be more sensitive in detecting strain-specific differences (17). There is one known species within the Dolosigranulum genus; however, our findings suggest the presence of more than one strain or species, particularly given that the $16 \mathrm{~S}$ rRNA V3/V4 region, as well as the wider $D$. pigrum genome, has been reported to be highly conserved (16). We did not see an inverse relationship with any of the main otopathogens, as described previously (18), which may be due to this population having a high baseline level of otopathogen colonization (5). There is growing interest in Dolosigranulum due to its association with URT and ear health; therefore, the use of whole-genome sequencing and ASV analysis will provide further understanding of the nuances of this nasal commensal.

Ornithobacterium was absent or was present with low relative abundance in the nasal microbiota of children with no history of OM. This likely represents "Candidatus Ornithobacterium hominis," a newly described species of Ornithobacterium that resides in the nasopharynx and is the only known human species in that genus (7). The role of "Candidatus Ornithobacterium hominis" in relation to respiratory/ear disease is still undetermined; however, it was originally found in Australian children and Thai refugee camp infants with high rates of respiratory disease $(19,20)$. Our findings suggest that Ornithobacterium may be associated with poor ear health. Furthermore, the network correlations supported relationships between Ornithobacterium, Helcococcus, and Dichelobacter, which may influence clinical outcomes. Intriguingly, the ASV and correlation network data suggest that there may be novel bacterial species within the nasal microbial ecosystem in genera that currently do not have human representatives (e.g., 

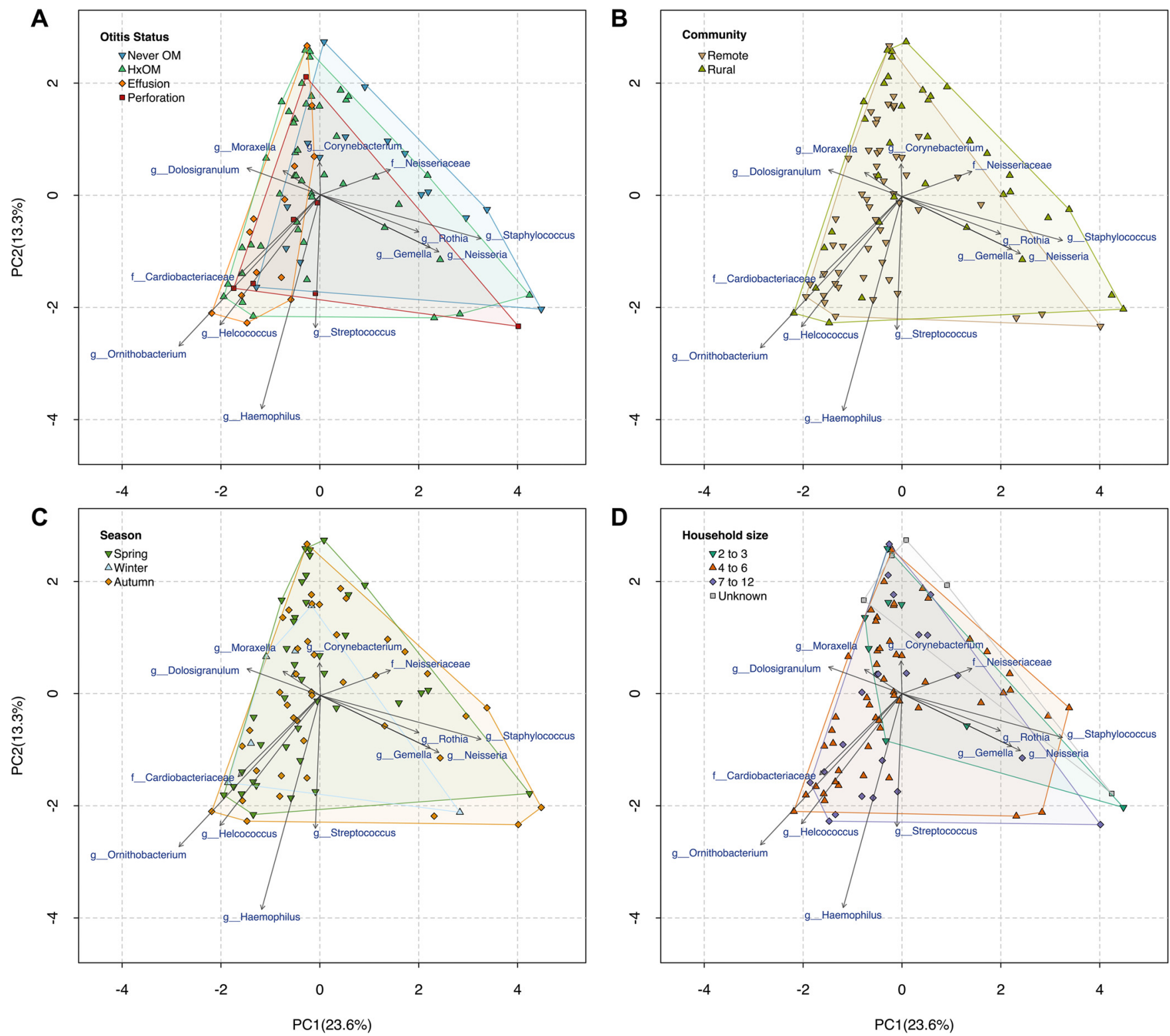

FIG 4 Genus-level PCA, showing the variation in microbial community composition across samples. Groups of samples are indicated by the colored hulls and points, with generally no separation being observed between groups according to OM status (A), community of residence (B), season of swab collection (C), and number of people residing in the household (D). A lack of separation between groups suggests that the microbial communities are largely similar between samples.

Dichelobacter and Gracilibacteria) or have only one species (Dolosigranulum). Along with Ornithobacterium, these genera warrant further investigations, particularly given their recurring relationships with genera associated with health and disease.

Although this is the largest NGS-based OM study in any Indigenous population to date, limitations exist. Recruitment and sample collection in remote Australian communities are resource- and time-intensive, which affected the sample size. Furthermore, recruitment of healthy children with no history of OM was challenging, despite our community-based sampling, reflecting the large burden of disease in remote Indigenous Australian communities (21). A well-powered, observational, prospective longitudinal birth cohort study would be ideal to elucidate the URT microbial ecology, interactions, and impacts on ear health among Aboriginal and Torres Strait Islander children and, given sufficient resources and time, should be achievable. We found that healthy children with no history of OM appeared to have differences in their nasal microbiota, depending on their community of 
residence; however, the small sample size limited further subgroup analysis. A well-recognized limitation of $16 \mathrm{~S}$ rRNA gene sequencing is its poor resolution at the species level. However, a combination of ASV and culturomic data partially overcame this limitation and provided novel species-level insights into the nasal microbial ecology in nose/ear health and disease. It is hoped that, moving forward, methods such as metagenomic shotgun sequencing can be optimized for the URT to provide a more comprehensive assessment of the URT microbiome in relation to health and disease.

In conclusion, our investigation of the nasal microbiota of Indigenous Australian children demonstrated that there is a potential synergism between $D$. pigrum and $C$. pseudodiphtheriticum that may be associated with ear and nose health and thus warrants further investigation. Our ASV-level analysis suggested that Dolosigranulum is a heterogeneous genus. Finally, we have detected the likely presence of "Candidatus Ornithobacterium hominis" and suggestions of other novel species within the nasal microbiota that are associated with poor URT/ear health.

\section{MATERIALS AND METHODS}

Additional details on the methods can be found in the supplemental material.

Population and sample collection. Indigenous Australian children 2 to 7 years of age were recruited prospectively from one rural community and one remote community in northern Queensland, Australia, in October 2015 to November 2017. Children who had received antibiotics within 3 weeks before sample collection were excluded (5). The study was approved by the Far North Queensland Human Research Ethics Committee (approval number HREC/15/QCH/10-594).

A detailed description of the cohort, sampling, and clinical data collection was documented previously (5). Briefly, demographic details and ear health history were collected for eligible children from parent interviews and the children's medical records. Children underwent ENT examinations (including otoscopy). Ear status at the time of swabbing was classified according to the more affected ear. Intranasal mucosal swab (dry FLOQSwabs; Copan Diagnostics, USA) samples used for molecular analysis were collected in parallel with Rayon swab (Transystem Minitip; Copan Diagnostics) samples for culturomics (5). All swab samples were kept at $4^{\circ} \mathrm{C}$ from the time of collection until arrival at the laboratory 24 to $48 \mathrm{~h}$ later. Molecular swab samples were then stored at $-80^{\circ} \mathrm{C}$.

DNA extraction and quality assurance. DNA was extracted via mechanical bead beating and tissue lysis, followed by automated MagNA Pure (Roche Diagnostics, Australia) extraction, as described previously (5). Four clean negative-control swabs were processed in parallel with the sample swabs. The quality of nasal sampling was assessed using a real-time PCR assay targeting the ERV3 marker for human DNA (8). Swab samples that were amplified with cycle thresholds of $\leq 38$ were considered to have adequate nasal epithelial cell content and, by extension, to be of good collection quality. Swab samples yielding cycle thresholds of $>38$ were excluded from further analysis.

16S rRNA gene sequencing. All sample and negative-control DNA extracts underwent 16S rRNA gene amplification using the 341F/806R primer set, followed by secondary indexing PCR. The equimolar library pool was then sequenced on a MiSeq instrument (Illumina, San Diego, CA, USA) with a 600-cycle v3 kit ( $2 \times 300$-bp paired-end reads).

Sequence data processing. Primer sequences were removed from demultiplexed reads using Cutadapt (v. 2.6). Reads were filtered and dereplicated using QIIME2 (v. 2019.10.0), and chimeras were removed by DADA2. Taxonomy was assigned to the resulting ASVs by aligning each (classify-consensusBLAST) against the nonredundant SILVA database (release 138).

Data analysis and statistics. Amplicon data analyses were performed in R (v. 4.0.2). ASVs that were not bacterial, fungal, or archaeal in origin, classified at or below the phylum level, or that were classified as chloroplast or mitochondrial were discarded. Putative contaminants were identified using decontam (v. 1.8.0) and microDecon (v. 1.0.2) and removed. ASVs with relative abundance of $<0.05 \%$ were also removed, and samples with less than 4,000 reads remaining were then discarded. Sample depth was limited to a maximum of 50,000 reads by using the rarefy function in vegan (v. 2.5-6).

Vegan was used to perform PCA, PERMANOVA, and PERMDISP on centered log-ratio-transformed ASV counts collapsed to the genus level. Differentially abundant ASVs and genera were identified using DESeq2 (v. 1.28.1). Alpha diversity metrics Chao1, Shannon, and Simpson were calculated using phyloseq (v. 1.32.0) on samples rarefied to 10,000 reads. Significant differences in alpha diversity distributions were determined with either Mann-Whitney $U$ tests or Kruskal-Wallis and Dunn's multiple-comparison tests, corrected for multiple testing using the Benjamini-Hochberg method. FastSpar (v. 0.0.10) was used for correlation analysis of genera.

Culturomic analysis. Culture-based swab samples were processed using an expanded agar protocol under aerobic and anaerobic conditions, with Vitek matrix-assisted laser desorption ionization-time of flight mass spectrometry (MALDI-TOF MS) (bioMérieux) isolate identification as described previously (5). Agreement between culture and 16S rRNA gene sequencing results was assessed using Cohen's kappa.

Data availability. Amplicon sequencing data have been deposited in the NCBI Sequence Read Archive (SRA) under BioProject number PRJNA684919, with GenBank accession numbers SRR13264782 to SRR13264885. 


\section{SUPPLEMENTAL MATERIAL}

Supplemental material is available online only.

SUPPLEMENTAL FILE 1, PDF file, 0.5 MB.

\section{ACKNOWLEDGMENTS}

We acknowledge the valuable contributions of Jason Leon, Chantel Hunter, Gail Wason, and Deborah Gertz from Mulungu Aboriginal Corporation Medical Centre, Isabel Toby from Save the Children, Doomadgee, and Anne O'Keefe from Doomadgee Community Health.

This work was supported by an Avant Doctors in Training Research Scholarship, a Queensland Health Junior Doctor Fellowship, and the University of Queensland Faculty of Medicine Strategic Funding. A. Coleman received support from an Australian National Health and Medical Research Council (NHMRC) Postgraduate Research Scholarship (grant APP1133366) and a Queensland Health Junior Doctor Fellowship. A. Cervin is supported by the University of Queensland Faculty of Medicine Strategic Funding and the Garnett Passe and Rodney Williams Memorial Foundation. S. Bialasiewicz is supported by NHMRC Program grants APP1071822 and APP1181054. The funders had no role in study design, data collection, interpretation, or the decision to submit the work for publication.

A. Coleman, A. Wood, E. G. Håkansson, J. Adams, M. Brown, A. Cervin, and S. Bialasiewicz designed the study. A. Coleman and A. Wood collected samples. A. Coleman, K. Cottrell, and S. Bialasiewicz conducted laboratory work. J. Zaugg and S. Bialasiewicz performed the bioinformatic analysis. A. Coleman, J. Zaugg, and S. Bialasiewicz wrote the manuscript. All authors revised and approved the final manuscript.

We declare no conflicts of interest.

\section{REFERENCES}

1. Monasta L, Ronfani L, Marchetti F, Montico M, Vecchi Brumatti L, Bavcar A, Grasso D, Barbiero C, Tamburlini G. 2012. Burden of disease caused by otitis media: systematic review and global estimates. PLoS One 7:e36226. https://doi.org/10.1371/journal.pone.0036226.

2. Kong K, Coates HLC. 2009. Natural history, definitions, risk factors and burden of otitis media. Med J Aust 191:S39-S43. https://doi.org/10.5694/j .1326-5377.2009.tb02925.x.

3. Coleman A, Wood A, Bialasiewicz S, Ware RS, Marsh RL, Cervin A. 2018. The unsolved problem of otitis media in indigenous populations: a systematic review of upper respiratory and middle ear microbiology in indigenous children with otitis media. Microbiome 6:199. https://doi.org/10 .1186/s40168-018-0577-2.

4. Jervis-Bardy J, Rogers GB, Morris PS, Smith-Vaughan HC, Nosworthy E, Leong LEX, Smith RJ, Weyrich LS, De Haan J, Carney AS, Leach AJ, O'Leary S, Marsh RL. 2015. The microbiome of otitis media with effusion in Indigenous Australian children. Int J Pediatr Otorhinolaryngol 79:1548-1555. https://doi.org/10.1016/j.jporl.2015.07.013.

5. Coleman A, Bialasiewicz S, Marsh RL, Grahn Håkansson E, Cottrell K, Wood A, Jayasundara N, Ware RS, Zaugg J, Sidjabat HE, Adams J, Ferguson J, Brown M, Roos K, Cervin A. 2021. Upper respiratory microbiota in relation to ear and nose health among Australian Aboriginal and Torres Strait Islander children. J Pediatric Infect Dis Soc 10:468-476. https://doi.org/10 .1093/jpids/piaa141.

6. Hugenholtz P. 2002. Exploring prokaryotic diversity in the genomic era. Genome Biol 3:reviews0003.1. https://doi.org/10.1186/gb-2002-3-2-reviews0003.

7. Salter S, Scott P, Page A, Tracey A, de Goffau M, Cormie C, OchoaMontaño B, Ling C, Tangmanakit J, Turner P, Parkhill J. 2019. "Candidatus Ornithobacterium hominis": insights gained from draft genomes obtained from nasopharyngeal swabs. Microb Genom 5:e000247. https:// doi.org/10.1099/mgen.0.000247.

8. Alsaleh A, Whiley D, Bialasiewicz S, Lambert S, Ware R, Nissen M, Sloots T, Grimwood K. 2014. Nasal swab samples and real-time polymerase chain reaction assays in community-based, longitudinal studies of respiratory viruses: the importance of sample integrity and quality control. BMC Infect Dis 14:15. https://doi.org/10.1186/1471-2334-14-15.

9. Teo SM, Tang HHF, Mok D, Judd LM, Watts SC, Pham K, Holt BJ, Kusel M, Serralha M, Troy N, Bochkov YA, Grindle K, Lemanske RF, Johnston SL,
Gern JE, Sly PD, Holt PG, Holt KE, Inouye M. 2018. Airway microbiota dynamics uncover a critical window for interplay of pathogenic bacteria and allergy in childhood respiratory disease. Cell Host Microbe 24:341-352. https://doi.org/10.1016/j.chom.2018.08.005.

10. Biesbroek G, Tsivtsivadze E, Sanders EAM, Montijn R, Veenhoven RH, Keijser BJF, Bogaert D. 2014. Early respiratory microbiota composition determines bacterial succession patterns and respiratory health in children. Am J Respir Crit Care Med 190:1283-1292. https://doi.org/10.1164/ rccm.201407-12400C.

11. Janek D, Zipperer A, Kulik A, Krismer B, Peschel A. 2016. High frequency and diversity of antimicrobial activities produced by nasal Staphylococcus strains against bacterial competitors. PLoS Pathog 12:e1005812. https:// doi.org/10.1371/journal.ppat.1005812.

12. Bosch AATM, de Steenhuijsen Piters W, van Houten $M$, Chu M, Biesbroek G, Kool J, Pernet P, de Groot P, Eijkemans M, Keijser B, Sanders E, Bogaert D. 2017. Maturation of the infant respiratory microbiota, environmental drivers, and health consequences: a prospective cohort study. Am J Respir Crit Care Med 196:1582-1590. https://doi.org/10.1164/rccm.201703-0554OC.

13. Pettigrew M, Laufer A, Gent J, Kong Y, Fennie K, Metlay J. 2012. Upper respiratory tract microbial communities, acute otitis media pathogens, and antibiotic use in healthy and sick children. Appl Environ Microbiol 78: 6262-6270. https://doi.org/10.1128/AEM.01051-12.

14. Lappan R, Imbrogno K, Sikazwe C, Anderson D, Mok D, Coates HL, Vijayasekaran S, Bumbak P, Blyth C, Jamieson S, Peacock C. 2018. A microbiome case-control study of recurrent acute otitis media identified potentially protective bacterial genera. BMC Microbiol 18:13. https://doi.org/10 .1186/s12866-018-1154-3.

15. Folino F, Fattizzo M, Ruggiero L, Oriano M, Aliberti S, Blasi F, Gaffuri M, Marchisio P, Torretta S. 2021. Nasopharyngeal microbiota analysis in healthy and otitis-prone children. Pediatr Infect Dis J 40:16-21. https:// doi.org/10.1097/INF.0000000000002895.

16. Brugger S, Eslami S, Pettigrew M, Escapa I, Henke M, Kong Y, Lemon K. 2020. Dolosigranulum pigrum cooperation and competition in human nasal microbiota. mSphere 5:e00852-20. https://doi.org/10.1128/mSphere.00852-20.

17. Callahan B, McMurdie P, Holmes S. 2017. Exact sequence variants should replace operational taxonomic units in marker-gene data analysis. ISME J 11:2639-2643. https://doi.org/10.1038/ismej.2017.119. 
18. Boelsen L, Dunne E, Mika M, Eggers S, Nguyen C, Tupou Ratu F, Russell F, Mulholland E, Hilty M, Satzke C. 2019. The association between pneumococcal vaccination, ethnicity, and the nasopharyngeal microbiota of children in Fiji. Microbiome 7:106. https://doi.org/10.1186/s40168-019-0716-4.

19. Marsh R, Kaestli M, Chang A, Binks M, Pope C, Hoffman L, Smith-Vaughan HC. 2016. The microbiota in bronchoalveolar lavage from young children with chronic lung disease includes taxa present in both the oropharynx and nasopharynx. Microbiome 4:37. https://doi.org/10.1186/s40168-016-0182-1.

20. Salter S, Turner C, Watthanaworawit W, de Goffau M, Wagner J, Parkhill J, Bentley S, Goldblatt D, Nosten F, Turner P. 2017. A longitudinal study of the infant nasopharyngeal microbiota: the effects of age, illness and antibiotic use in a cohort of South East Asian children. PLoS Negl Trop Dis 11: e0005975. https://doi.org/10.1371/journal.pntd.0005975.

21. Morris P, Leach AJ, Silberberg P, Mellon G, Wilson C, Hamilton E, Beissbarth J. 2005. Otitis media in young Aboriginal children from remote communities in northern and central Australia: a cross-sectional survey. BMC Pediatr 5:27. https://doi.org/10.1186/1471-2431-5-27.

22. Australian Technical Advisory Group of Immunisation. 2017. The Australian immunisation handbook. Australian Government Department of Health, Canberra, Australia. 